Nuevo registro de ocelote en el Parque

Ecológico Chipinque, Nuevo León, México

\title{
A new record of ocelot in Parque Ecológico Chipinque, Nuevo León, México
}

\author{
Margarita García-Bastida ${ }^{1}{ }^{*}$, Francisco Martínez-de la Fuente ${ }^{1}$, Alicia Vázquez-Venegas ${ }^{1}$ y Juan L. Peña-Mondragón² .
}

\author{
${ }^{1}$ Departamento de Investigación y Manejo de Recursos Naturales, Parque Ecológico Chipinque, A.B.P. Carretera a Chipinque Km \\ 2.5 s/n. San Pedro Garza García, 66290, Nuevo León. México. E-mail: magaba28@hotmail.com (MGB), fmar.fte@gmail.com (FMF), \\ avazquez@chipinque.org.mx (AVV). \\ ${ }^{2}$ Instituto de Investigaciones en Ecosistemas y Sustentabilidad (IIES), UNAM-Campus Morelia, Antigua Carretera a Pátzcuaro 8701, \\ Morelia 58190, Michoacán, México. E-mail: jlpena@cieco.unam.mx (JLPM) \\ ${ }^{*}$ Corresponding author
}

The ocelot (Leopardus pardalis) is one of the six felid species recorded in Mexico, and it is present in diverse habitats. In Mexico it is listed as endangered (NOM-059, SEMARNAT-2010), but, information about its populations is limited, especially in Nuevo Léon. Habitat loss is an important factor which jeopardizes ocelot survival and conservation efforts in private protected areas are important for preserving this species. Parque Ecológico Chipinque (PECh) is a private natural area in the Sierra Madre Oriental (Subprovince Gran Sierra Plegada), and is part of a large scale Parque Nacional Cumbres de Monterrey, a Natural Protected Area, in Nuevo León, México. In 2010, as part of "Biodiversity Monitoring", PECh implemented a "Mammal Monitoring Program" establishing 11 monitoring stations with cam traps located in pine-oak forest, oak-pine forest, oak forest and piedmont scrub throughout the park and separated each other 0.9 to $2 \mathrm{~km}$. The main goal of this program is to register mammal species previously listed for PECh and species with potential distribution range in the area. Parque Ecológico Chipinque is located in the potential distribution range of the ocelot, and mammal monitoring program implemented in PECh has been successful: two events from ocelot (Leopardus pardalis) were obtained with cam traps in September 2014 in a pine-oak $(1,274 \mathrm{~m})$ and oak-pine $(1,390 \mathrm{~m})$ forest. These new records are very important in confirming the continuing presence of ocelot in its potential distribution range in the Sierra Madre Oriental. The presence of ocelot (Leopardus pardalis) in Parque Ecológico Chipinque suggests the probability that the park serves as a biological corridor for the species, and also suggests the health of the forest conserved in this area. Parque Ecológico Chipinque as a private protected area is playing an important role in species conservation, despite its tourism use and closeness to the urban zone.

El ocelote (Leopardus pardalis) es una de las seis especies de félidos enlistadas para México y se ha registrado en diferentes hábitats. En México esta enlistado como especie en peligro de extinción (NOM-059, SEMARNAT-2010), a pesar de esto la información acerca de sus poblaciones es limitada, principalmente en Nuevo León. Actualmente la pérdida del hábitat es uno de los principales factores que pone en riesgo la presencia del ocelote y los esfuerzos de conservación realizados por las áreas protegidas privadas son importantes para preservar esta especie. El Parque Ecológico Chipinque (PECh) es un área natural privada ubicada en la Sierra Madre Oriental (Subprovincia Gran Sierra Plegada) y forma parte del Área Natural Protegida Parque Nacional Cumbres de Monterrey, en Nuevo León, México. En el 2010 como parte del "Programa de Monitoreo de la biodiversidad", se implementó el programa "Monitoreo de mastofauna" en el PECh, para lo cual se establecieron 11 estaciones de monitoreo con cámaras-trampa ubicadas en bosque de pino-encino, bosque de encino-pino, bosque de encino y matorral submontano, separadas entre sí de 0.9 a $2 \mathrm{~km}$. El principal objetivo de este programa es registrar especies de mamíferos previamente listadas y especies con potencial de distribución en el área del PECh. El Parque Ecológico Chipinque se localiza en el área de distribución potencial del ocelote (Leopardus pardalis) y el programa implementado de monitoreo de mamíferos ha sido exitoso: se ha obtenido el registro de individuos adultos en dos eventos independientes capturados con cámarastrampa en septiembre de 2014 en un bosque de pino-encino $(1,274 \mathrm{~m})$ y el otro en bosque de encino-pino (1,390 m). Este nuevo registro es de alta importancia y confirma la presencia del ocelote en su área de distribución potencial en la Sierra Madre Oriental. La presencia del ocelote (Leopardus pardalis) en el Parque Ecológico Chipinque sugiere la probabilidad de que este sirva como un corredor biológico de la especie, además de sugerir el buen estado de la vegetación del sitio. De este modo, el Parque Ecológico Chipinque como área protegida privada está desempeñando su papel en la conservación de especies a pesar de ser un área de uso turístico y de su proximidad con la zona urbana.

Key words: conservation; Felidae; Leopardus pardalis.

๑) 2016 Asociación Mexicana de Mastozoología, www.mastozoologiamexicana.org 


\section{Introducción}

El ocelote Leopardus pardalis es uno de los seis felinos reportados para México (Ceballos y Oliva 2005); es de talla mediana (adultos pesan entre 6 y 15 kilogramos) y la cola es proporcionalmente corta; el color de su cuerpo va de café a café grisáceo, con un patrón de manchas alargadas en los costados; es parecido al margay (Leopardus wiedii), pero este es de menor tamaño (adultos pesan entre 3 y 7 kilos), con grandes ojos y cola larga (Murray y Gardner 1997; Aranda 2012). El ocelote habita en zonas tropicales y áreas de transición con zonas neárticas; se ha registrado en selva baja caducifolia (Martínez-Calderas et al. 2011; Valdez-Jiménez et al. 2013), bosque de oyamel (Aranda et al. 2014), bosque de encino, bosque tropical Iluvioso (Almazán-Catalán et al. 2013; Pérez-Irineo y Santos-Moreno 2014), matorral submontano, selva mediana, selva alta perennifolia, bosque mesófilo de montaña, bosque de pino-encino y matorral desértico micrófilo (Martínez-Calderas et al. 2011; Almazán-Catalán et al. 2013) en altitudes hasta los 3,150 m (Almazán-Catalán et al. 2013; Aranda et al.2014). Su distribución está registrada desde el sureste de Estados Unidos hasta el norte de Argentina (Murray y Gardner 1997). En México se ha registrado desde Sonora hasta Chiapas y desde Nuevo León y Tamaulipas hasta Quintana Roo (Grigione et al. 2009; CONABIO 2010).

En México la especie se encuentra catalogada "en peligro de extinción" (SEMARNAT 2010), mientras que a nivel internacional como "de preocupación menor" (IUCN 2015) y su comercialización se encuentra regulada por CITES, donde se ubica en el Apéndice I (CITES 2015). Actualmente el comercio ilegal representa una amenaza para la especie, en conjunto con la pérdida y fragmentación del hábitat y la matanza en represalia por la depredación de aves de corral (Caso et al. 2008).

El conocimiento sobre la distribución del ocelote en Nuevo León se limita a siete registros documentados: un ejemplar colectado en abril de 1946 cerca del municipio de General Bravo (Jiménez-Guzmán et al. 1999), dos ejemplares cazados: uno en 1940 en el municipio de Santiago (Moreno-Valdéz 1998) y otro en 1995 en el municipio de China (Jiménez-Guzmán et al. 1999); tres reportes como fauna asociada al jaguar en la Gran Sierra Plegada (Sierra Madre Oriental), en vegetación de matorral submontano y bosque de encino (Rosas-Rosas y López-Soto 2002) y el registro más reciente es un ejemplar encontrado muerto en el Área Natural Protegida Cerro de la Silla, en el municipio metropolitano de Guadalupe, en vegetación de matorral submontano y bosque de encino (Velazco-Macías y Peña-Mondragón 2015; Figura 1a).

\section{Materiales y métodos}

El Parque Ecológico Chipinque (PECh) es un Área Natural Protegida privada ubicada en el límite noreste del Área Natural Protegida Federal "Parque Nacional Cumbres de Monterrey" (PNCM), Nuevo León, México y su uso principal es la conservación. Colinda al norte con el área metropolitana de Monterrey; al este con vegetación de bosque templado; al sur con matorral desértico rosetófilo y al oeste con bosque templado y un fraccionamiento habitacional de baja densidad. A aproximadamente $9 \mathrm{~km}$ en dirección oeste-noroeste la vegetación cambia de bosque templado a matorral submontano. Como parte de un estudio de "Monitoreo de la mastofauna" implementado en el PECh desde agosto de 2010 se establecieron 11 estaciones de cámaras-trampa (Bushnell; https://www.bushnell.mx; simples, sin cebo ni atrayentes) espaciadas entre sí de 0.9 a 2 km en bosque de pino-encino, bosque de encino-pino, bosque de encino y matorral submontano (siendo las especies representativas en bosque Pinus pseudostrobus, P. teocote, Quercus rysophilla, Q. canbyi, Q. polymorpha, mientras que en matorral submontano son Helietta parvifolia, Cordia boissieri, Pithecellobium pallens; Figura 1b). 


\section{Resultados}

Desde que se colocaron las estaciones de cámaras-trampa, estas han permanecido encendidas permanentemente y a la fecha se han obtenido dos fotografías de ocelotes adultos en eventos independientes. El primer evento se registró el 2 de septiembre de 2014 a las 5:50 h (25 35'37.05" $\mathrm{N},-100^{\circ} 19^{\prime} 46.55^{\prime \prime} \mathrm{O} ; 1,274 \mathrm{~m}$; bosque de pino-encino; Figura 1b). El segundo evento se registró el 14 de septiembre a las 5:28 h ( $25^{\circ} 36^{\prime} 14.79^{\prime \prime} \mathrm{N},-100^{\circ} 21^{\prime} 14.36^{\prime \prime} \mathrm{O} ; 1,390 \mathrm{~m}$; bosque de encino-pino; Figuras 2a, 2b); el área se caracteriza por ser terreno abrupto con altas pendientes. Para corroborar la identificación de la especie se utilizó el método de triple ciego (Kelly et al. 2008).

Para hacer la colocación de las cámaras-trampa se utilizó como referencia el tamaño del ámbito hogareño del gato cola rabona ( Lynx rufus, $1.12 \mathrm{Km}^{2}$ ), por ser de hábitos alimenticios estrictamente carnívoros y ser el felino de menor movilidad presente en el PECh. Las estaciones en las que se registró el ocelote se encuentran separadas entre sí $2.6 \mathrm{~km}$ y están ubicadas a $3 \mathrm{~km}$ y 0.4 $\mathrm{km}$ de la principal zona de recreación del PECh; ambos sitios son veredas poco transitadas por visitantes. Según Grigione et al. 2009 el Parque Ecológico Chipinque se encuentra dentro del área de distribución potencial del ocelote, por lo que con este primer registro del ocelote en el PECh se confirma su presencia.

\section{Discusión}

Las cámaras-trampa son una técnica eficiente en la búsqueda de nuevos registros de especies elusivas y de hábitos crípticos, como el ocelote. Estudios previos muestran que la distribución del ocelote va desde bosques de coníferas y matorral hasta bosques tropicales. Se ha sugerido que la presencia de la especie está restringida por factores como el estado de conservación del hábitat (Haines et al. 2006) y características abióticas del sitio como la temperatura y la precipitación (Jiménez-Maldonado 2007).

Es notable la falta de estudios sobre la distribución del ocelote en Nuevo León; previamente esta especie ha sido reportada en el norte del estado en los municipios de General Bravo (JiménezGuzmán et al. 1999), Santiago (Moreno-Valdéz 1998), China (Jiménez-Guzmán et al. 1999); en la Gran Sierra Plegada (Rosas-Rosas y López-Soto 2002, sin especificar municipios) y Guadalupe (Velazco-Macías y Peña-Mondragón 2015). Los reportes realizados sobre la distribución en el noreste de México sugieren que es posible que la Gran Sierra Plegada albergue una población
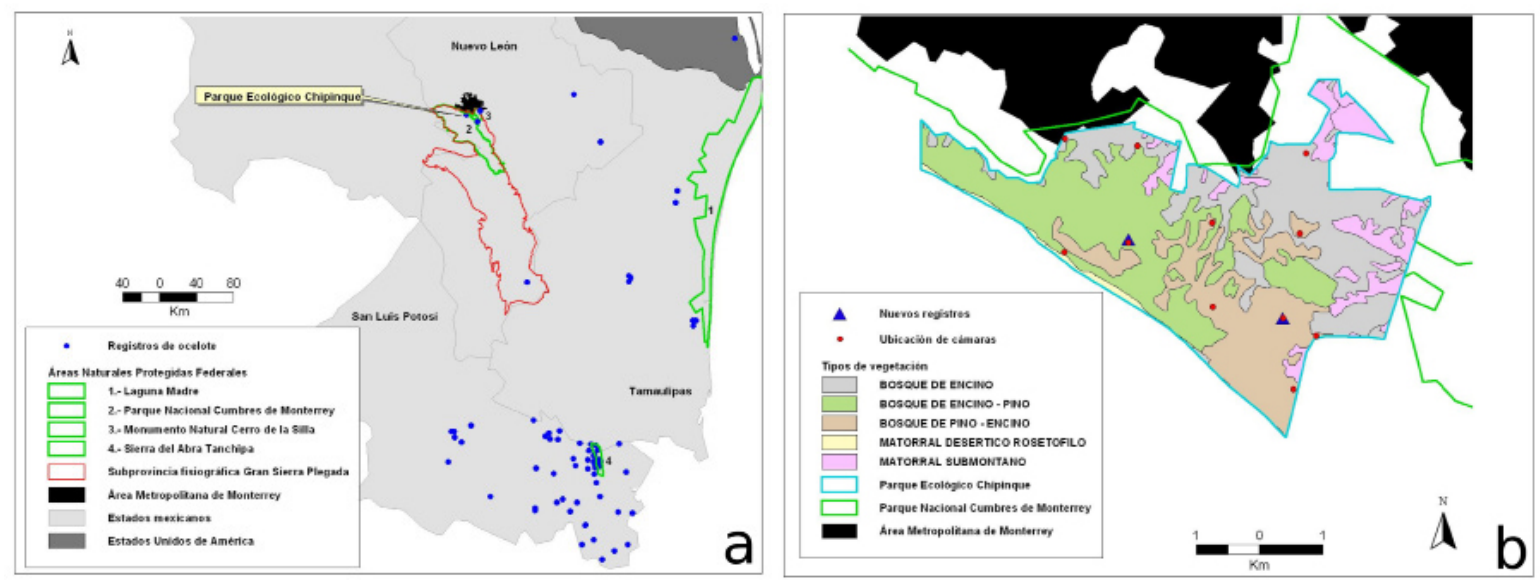

Figura 1. A) Registros de ocelote en el noreste de México (obtenidos de literatura) y registros nuevos en el Parque Ecológico Chipinque, donde se muestra la ubicación del Parque en relación con la Subprovincia Fisiográfica Gran Sierra Plegada. B. Ubicación de las cámaras trampa en el Parque Ecológico Chipinque, señalando los nuevos registros de ocelote y los tipos de vegetación. 


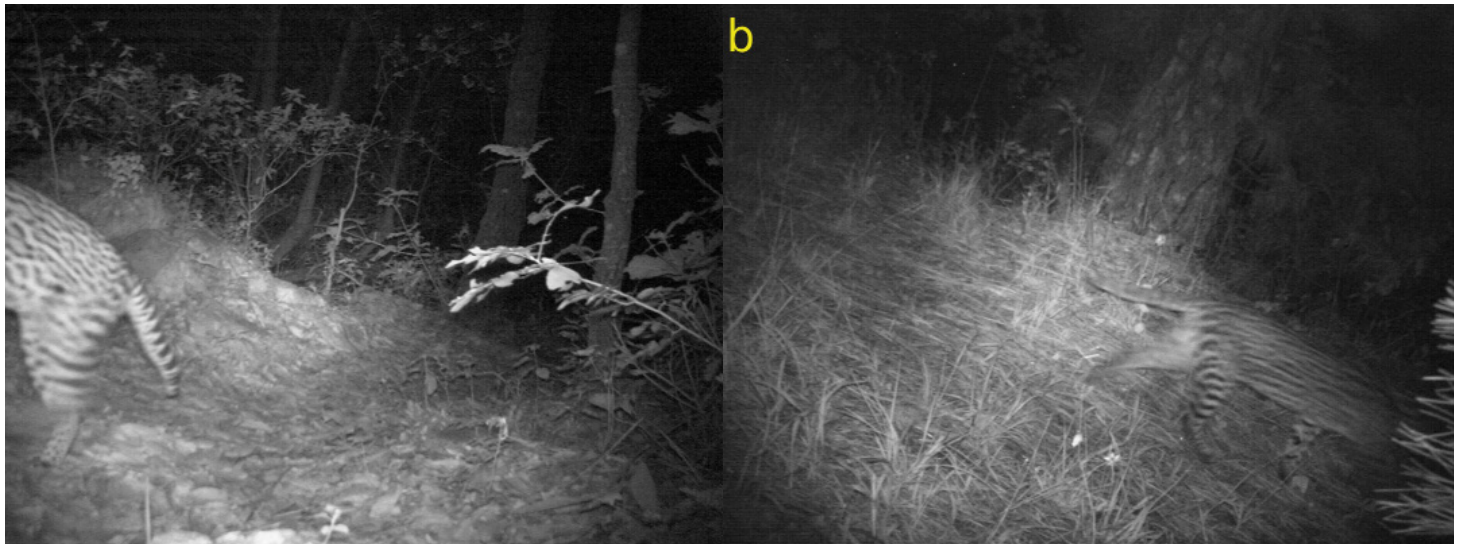

Figura 2. Registros de ocelote en bosque de pino-encino (1,274 m) el 2 de septiembre de 2014 a las 5:50 h. B. Registro de ocelote en bosque de encino-pino (1,390 m) el 14 de septiembre a las 5:28 $\mathrm{h}$.

residente de ocelote que conecta las poblaciones del norte con las del sur. El Parque Ecológico Chipinque (PECh) se encuentra dentro de la gran Sierra Plegada (Figura 1a) y, de acuerdo con otros autores, debido a las características de vegetación (Rosas-Rosas y López-Soto 2002; Ramírez Bravo et al. 2010; Martínez-Calderas et al. 2011), grado de conservación (Haines et al. 2006), altitud (Almazán-Catalán et al. 2013; Aranda et al. 2014) y características abióticas de temperatura y precipitación (Jiménez-Maldonado 2007) que se presentan en el PECh, es probable que este sea parte del área que sirve como corredor biológico para la especie. En Estados Unidos las únicas poblaciones conocidas de ocelote se limitan al extremo sur-este de Texas, donde la propiedad privada constituye el $97 \%$ de su territorio y por tanto la conservación depende fuertemente del apoyo de sus propietarios (Haines et al. 2006). Dentro del Parque Nacional Cumbres de Monterrey el Parque Ecológico Chipinque es un caso especial de propiedad privada enfocada al turismo y destaca por su importancia como zona de conservación y de resguardo de especies amenazadas y sensibles al disturbio causado por factores antropogénicos (e. g. oso negro, puma y jaguaroundi).

\section{Agradecimientos}

Los autores quieren agradecer al personal del Parque Ecológico Chipinque, en especial a la Lic. L. Willcockson, directora del PECh por el apoyo otorgado a este proyecto.

\section{Literatura citada}

Almazán-Catalán, J. A, C. Sánchez-Hernández, F. Ruíz-Gutiérrez, M. L. Romero-Almaraz, A. Taboada-Salgado, E. Beltrán-Sánchez, y L. Sánchez-VÁzquez. 2013. Registros adicionales de felinos del estado de Guerrero, México. Revista Mexicana de Biodiversidad 84:347-359.

Aranda, S. J. M. 2012. Manual para el rastreo de mamíferos silvestres de México. Comisión Nacional para el Conocimiento y Uso de la Biodiversidad. Ciudad de México, México.

Aranda, M., F. Botello, E. Martínez-Meyer, y A. Pineda. 2014. Primer registro de ocelote (Leopardus pardalis) en el Parque Nacional Lagunas de Zempoala, Estado de México y Morelos, México. Revista Mexicana de Biodiversidad 85:1300-1302.

Caso, A. C. Lopez-Gonzalez, E. Payan, E. Eizirik, T. de Oliveira, R. Leite-Pitman, M. Kelly, y C. Valderrama. 2008. Leopardus pardalis. The IUCN Red List of Threatened Species. Version 2014.2. www.iucnredlist. org. Última visita 20 marzo 2015.

Ceballos, G., y G. Oliva (Eds.). 2005. Los mamíferos silvestres de México. Comisión para el Conocimiento y Uso de la Biodiversidad. Fondo de Cultura Económica. Ciudad de México, México. 
Convención sobre el Comercio Internacional de Especies Amenazadas de Fauna y Flora Silvestres (CITES). 2015. Convención sobre el Comercio Internacional de Especies Amenazadas de Fauna y Flora Silvestres (CITES). Apéndices I, II y III en vigor a partir del 24 de julio de 2010. http://www.cites.org/. Última visita: 30 marzo 2015.

Comisión Nacional para el Conocimiento y uso de la Biodiversidad (CONABIO). 2010. http:// www.conabio.gob.mx/informacion/gis/layouts/leopar_dcgw.png. Última visita: 30 marzo 2015.

Grigione, M. M., K. Menke, C. López-Gonzalez, R. List, A. Banda, J. Carrera, R. Carrera, A. J. Giordano, J. Morrison, M. Sternberg , R Thomas, y B. Van Pelt. 2009. Identifying potential conservation areas for felids in the USA and Mexico: integrating reliable knowledge across an international border. Fauna and Flora International. Oryx 43:78-86.

Haines, A. M., J. E. Janecka, M .E. Tewes, L. I. Grassman, y P. Morton. 2006. The importance of private lands for ocelot Leopardus pardalis conservation in the United States. Oryx 40:90-94.

International Union for Conservation of Nature (UICN). 2015. The IUCN red list of threatened species. Version 2014.3. http://www.iucnredlist.org. Última visita: 6 enero 2015.

Jiménez-Guzmán, A., M. A. Zuñiga-Ramos, y J. A. Niño-Ramírez. 1999. Mamíferos de Nuevo León (En línea). http://www.fcb.uanl.mx/mastozoología/. Última visita 20 de marzo de 2015.

Jiménez-Maldonado, R. E. 2007. Modelación de nicho ecológico y coexistencia de ocelotes (Leopardus pardalis) y gatos monteses (Lynx rufus) en México (Tesis de maestría). Universidad Autónoma de Querétaro. México.

Kelly, M. J., A. J. Noss, M. S. Di Bitettı, L. Maffei, R. L. Arispe, A. Paviolo, C.D. de Angelo, y Y. E. DI BLANCo. 2008. Estimating puma densities from camera trapping across three study sites: Bolivia, Argentina, and Belize. Journal of Mammalogy 89:408-418.

Martínez-Calderas J. M., O. C. Rosas-Rosas, J. F. Martínez-Montoya, L. A Tarango-Arámbula, F. Clemente-Sánchez, M. M. Crosby-Galván y M. D. Sánchez-Hermosillo. 2011. Distribución del ocelote (Leopardus pardalis) en San Luis Potosí, México. Revista Mexicana de Biodiversidad 82:997-1004.

Moreno-Valdéz, A. 1998. Mamíferos del Cañón de Huajuco, municipio de Santiago, Nuevo León, México. Revista Mexicana de Mastozoología 3:5-25.

MurRaY, J. L., y G. L. Gardner. 1997. Leopardus pardalis. Mammalian species 548:1-10.

Pérez-Irineo, G., y A. Santos- Moreno. 2014. Density, distribution, and activity of the ocelot Leopardus pardalis (Carnívora: Felidae) in Southeast Mexican rainforest. Revista de Biología Tropical 62:1421-1432.

Ramírez-Bravo O. E., E. Bravo-Carrete, C. Hernández-Santín, S. Schinkel-Brault y K. Chris. 2010. Ocelot (Leopardus pardalis) distribution in the state of Puebla, Central Mexico. Therya 1:111-120.

Rosas- Rosas, O. C., y J. H. López-Soto. 2002. Distribución y estado de conservación del jaguar en Nuevo León, México. Pp. 393-401 en El jaguar en el nuevo milenio (Medellín, R. A., C. Equihua, C. L. B. Chetkiewicz, G. Crawshaw Jr, A. Rabinowitz, K. H. Redford, J. G. Robinson, E. W. Sanderson, y A. B. Taber, eds.). Fondo de Cultura Económica, Universidad Nacional Autónoma de México y Wildlife Conservation Society. Ciudad de México, México.

Secretaría de Medio Ambiente y Recursos Naturales. 2010. Norma Oficial Mexicana NOM059- SEMARNAT -2010, Protección Ambiental -Especies de flora y fauna silvestres en México - Categorías de riesgo y especificaciones para su inclusión, exclusión o cambio, Lista de especies en riesgo. Diario Oficial de la Federación (DOF). Ciudad de México, México.http://dof.gob.mx/nota detalle.php?codigo=5173091\&fecha=30/12/2010. Última visita: 6 enero 2015. 
Valdez-Jiménez, D., C. M. García-Balderas, y G. E. Quintero-Díaz. 2013. Presencia del ocelote (Leopardus pardalis) en la "Sierra del Laurel", Municipio de Calvillo, Aguascalientes, México. Acta Zoológica Mexicana (n. s.) 29:688-692.

Velazco-Macías, C. G. y J. L. Peña-Mondragón. 2015. Nuevo registro de ocelote (Leopardus pardalis) en el estado de Nuevo León, México. Acta Zoológica Mexicana (n. s.) 31:451453.

Submited: April 27, 2015

Reviewed: July 22, 2015

Accepted: November 4, 2015

Associated editor: Rafael Reyna 